\title{
Nonexistence results for tight block designs
}

\author{
Peter Dukes · Jesse Short-Gershman
}

Received: 16 October 2011 / Accepted: 28 August 2012 / Published online: 14 September 2012

(C) Springer Science+Business Media, LLC 2012

\begin{abstract}
Recall that combinatorial $2 s$-designs admit a classical lower bound $b \geq\left(\begin{array}{l}v \\ s\end{array}\right)$ on their number of blocks, and that a design meeting this bound is called tight. A long-standing result of Bannai is that there exist only finitely many nontrivial tight $2 s$-designs for each fixed $s \geq 5$, although no concrete understanding of 'finitely many' is given. Here, we use the Smith Bound on approximate polynomial zeros to quantify this asymptotic nonexistence. Then, we outline and employ a computer search over the remaining parameter sets to establish (as expected) that there are in fact no such designs for $5 \leq s \leq 9$, although the same analysis could in principle be extended to larger $s$. Additionally, we obtain strong necessary conditions for existence in the difficult case $s=4$.
\end{abstract}

Keywords Tight design - Symmetric design · Orthogonal polynomials ·

Delsarte theory

\section{Introduction}

Let $v \geq k \geq t$ be positive integers and $\lambda$ be a nonnegative integer. A $t-(v, k, \lambda)$ design, or simply a $t$-design, is a pair $(V, \mathcal{B})$ where $V$ is a $v$-set and $\mathcal{B}$ is a collection of $k$ subsets of $V$ such that any $t$-subset of $V$ is contained in exactly $\lambda$ elements of $\mathcal{B}$. The elements of $V$ are points and the elements of $\mathcal{B}$ are blocks. Since $t$-designs are also $i$-designs for $i \leq t$, the parameter $t$ is typically called the strength of the design (when it is as large as possible). The number of blocks is usually denoted $b$ and an easy double-counting argument shows $b=\lambda\left(\begin{array}{l}v \\ t\end{array}\right) /\left(\begin{array}{l}k \\ t\end{array}\right)$.

P. Dukes $(\bowtie) \cdot$ J. Short-Gershman

Mathematics and Statistics, University of Victoria, Victoria, BC V8W 3R4, Canada

e-mail: dukes@uvic.ca

J. Short-Gershman

e-mail: jesseasg@uvic.ca 
Suppose $(V, \mathcal{B})$ is a $t-(v, k, \lambda)$ design. Generalizing Fisher's Inequality, RayChaudhuri and Wilson [10] showed that if $t$ is even, say $t=2 s$, and $v \geq k+s$, then $b \geq\left(\begin{array}{l}v \\ s\end{array}\right)$. If equality holds in this bound, we say $(V, \mathcal{B})$ is tight. The trivial tight $2 s$-designs are those with $v=k+s$, where each of the $\left(\begin{array}{l}v \\ k\end{array}\right)=\left(\begin{array}{l}v \\ s\end{array}\right) k$-subsets of $V$ is a block. The case of odd strength is investigated in [5]; however, it is impossible for $(2 s-1)$-designs to be tight in the sense of having $\left(\begin{array}{c}v \\ s-1\end{array}\right)$ blocks.

Returning to even strength, the full set of parameters $v$ and $k$ for which a tight $2 s$ design exists has only been determined for $s=2,3$. Note that, when $s=1$, tight 2designs have $b=v$ and are the 'symmetric' designs; see [6,8] for surveys of this rich (yet very challenging) topic. In 1975, Ito [7] published a proof that the only nontrivial tight 4-designs are the Witt 4-(23,7,1) design and its complementary 4-(23,16,52) design, but his proof was found to be incorrect. A few years later, Enomoto, Ito, and Noda [4] proved the weaker result that there are finitely many nontrivial tight 4designs, though still believing Ito's initial claim to be true. Finally, in 1978, Bremner [2] successfully settled $s=2$ by reaffirming Ito's result. Peterson [9] proved in 1976 that there exist no nontrivial tight 6-designs.

Bannai [1] proved that there exist only finitely many nontrivial tight $2 s$-designs for each $s \geq 5$. The case $s=4$ is quite open, and the 'finitely many' for $s \geq 5$ is not explicit and potentially grows with $s$. However, it is probably the case that there are no unknown tight $2 s$-designs for $s \geq 2$.

Central to these negative results is the concept of the intersection numbers of a design. An integer $0 \leq \mu<k$ is an intersection number of a $t-(v, k, \lambda) \operatorname{design}(V, \mathcal{B})$ if there exist different blocks $B_{1}, B_{2} \in \mathcal{B}$ such that $\left|B_{1} \cap B_{2}\right|=\mu$. The following strong condition was discovered first by Ray-Chaudhuri and Wilson [10] and also implicitly by Delsarte [3].

Proposition $1.1([3,10])$ If there exists a tight $2 s-(v, k, \lambda)$ design, then the zeros of the following degree s polynomial $\Psi_{s}(x)$ are the intersection numbers of the design, and hence they must all be nonnegative integers:

$$
\Psi_{S}(x)=\sum_{i=0}^{s}(-1)^{s-i} \frac{\left(\begin{array}{c}
v-s \\
i
\end{array}\right)\left(\begin{array}{c}
k-i \\
s-i
\end{array}\right)\left(\begin{array}{c}
k-1-i \\
s-i
\end{array}\right)}{\left(\begin{array}{c}
s \\
i
\end{array}\right)}\left(\begin{array}{l}
x \\
i
\end{array}\right) .
$$

The polynomials $\Psi_{s}$ are known as the Gegenbauer polynomials.

Since a $2 s$-design with $v \geq k+s$ induces at least $s$ intersection numbers [10], it follows that the zeros of $\Psi_{s}$ must additionally be distinct integers for tight designs. Note also that $\Psi_{s}$ has no dependence on $\lambda$; indeed, for tight designs $\lambda=\left(\begin{array}{c}v \\ s\end{array}\right)\left(\begin{array}{c}k \\ 2 s\end{array}\right) /\left(\begin{array}{c}v \\ 2 s\end{array}\right)$ and is therefore uniquely determined by $v$ and $k$.

Analogously, the Lloyd polynomials $L_{e}(x)$ are important for the characterization of perfect $e$-error-correcting codes; see [13]. It is interesting that this characterization of perfect codes was completed long ago, while the open problems mentioned before Proposition 1.1 remain for tight designs. Our goal here is to revive the interest in tight designs and take a modest step toward the full characterization of their parameters.

The outline is as follows. In Sect. 2, we review the work of Bannai in [1] on the asymptotic structure of the zeros of $\Psi_{s}$. Extending this, we obtain some exact bounds relevant to this analysis. Section 3 summarizes the techniques for exhausting small 
cases $s \geq 5$, and Sect. 4 is devoted to a partial analysis of the case $s=4$. An appendix of tables following the main text will prove useful to the interested reader.

\section{Bannai's analysis and the Smith bound}

\subsection{Notation}

Assuming a tight design, let $x_{i}$, for $i=-\left\lfloor\frac{s}{2}\right\rfloor, \ldots,(0), \ldots,\left\lfloor\frac{s}{2}\right\rfloor$, denote the zeros of $\Psi_{s}$ listed in increasing order. For example, the zeros of $\Psi_{4}$ and $\Psi_{5}$ are denoted $x_{-2}<x_{-1}<x_{1}<x_{2}$ and $x_{-2}<x_{-1}<x_{0}<x_{1}<x_{2}$, respectively.

An important parameter is the arithmetic mean of the zeros of $\Psi_{S}(x)$, which we will denote by $\bar{\alpha}$. From the coefficient of $x^{s-1}$, we have

$$
\bar{\alpha}=\frac{(k-s+1)(k-s)}{v-2 s+1}+\frac{s-1}{2} .
$$

It will be useful as in [1] to define the parameter $\alpha$ so that $\bar{\alpha}=\alpha+(s-1) / 2$, that is,

$$
\alpha=\frac{(k-s+1)(k-s)}{v-2 s+1} .
$$

We also define

$$
\tau=\frac{v-2 s+1}{k-s+1}
$$

Bannai [1] denoted this quantity by $t$, but we feel $t$ should be reserved for the strength of the design.

Note $\tau=2$ implies $v=2 k+1$. Moreover, if $v<2 k$, we may complement blocks, replacing $k$ with $v-k$ and obtain $v>2 k$. This is discussed further in Sect. 2.2.

Finally, put $\beta=\left(1-\frac{1}{\tau}\right) \sqrt{\alpha}$. In terms of $v$ and $k$,

$$
\beta=\frac{(v-k-s) \sqrt{(k-s+1)(k-s)}}{(v-2 s+1)^{3 / 2}} .
$$

In particular, $\beta=0$ if and only if $v=k+s$, so in some sense $\beta$ can be seen as measuring the 'nontriviality' of a (tight) $2 s$-design. Note also that

$$
\begin{aligned}
& k=\tau^{3}(\tau-1)^{-2} \beta^{2}+s, \quad \text { and } \\
& v=\tau^{4}(\tau-1)^{-2} \beta^{2}+\tau+2 s-1 .
\end{aligned}
$$

Bannai's proof of the existence of only finitely many nontrivial tight $2 s$-designs, $s \geq 5$, is divided into cases according to this parameter $\beta$. In particular, he proves

- for any $\beta_{0}$, there are only finitely many tight $2 s$-designs with $\beta \leq \beta_{0}$; and

- there exists $\beta_{0}$ (depending only on $s$ ), such that there are no nontrivial tight $2 s$ designs with $\beta>\beta_{0}$.

Here, our main goal is to compute such a $\beta_{0}$ explicitly for $5 \leq s \leq 9$ and, by searching across all pairs $(v, k)$ for which $\beta \leq \beta_{0}$, show that there are in fact zero nontrivial tight $2 s$-designs for these $s$. 


\subsection{Symmetry with respect to the parameter $\tau$}

In the analytic work which follows, it is helpful to obtain a lower bound on $\tau$. As discussed above, we may complement blocks to assume $v \geq 2 k$. The following was mentioned but not fully proven in [1].

Lemma 2.1 Let $s \geq 1$. There are no tight $2 s$-designs with $v=2 k$.

Proof Suppose $v=2 k$. Then from (2.1), $s \bar{\alpha}-\left(\begin{array}{l}s \\ 2\end{array}\right)=s \alpha=\frac{s(k-s+1)(k-s)}{2(k-s)+1}$. Without too much effort, it can be seen that the least residue of $s(k-s+1)(k-s)(\bmod 2(k-$ $s)+1$ ), denoted here by $r_{k, s}$, satisfies

$$
r_{k, s}= \begin{cases}2(k-s)-\frac{s-4}{4} & \text { if } s \equiv 0(\bmod 4) \\ k-s-\frac{s-2}{4} & \text { if } s \equiv 2(\bmod 4) \\ \frac{k-s}{2}-\frac{s-1}{4} & \text { if } s \equiv 1(\bmod 4) \text { and } k \text { is odd } \\ & \text { or } s \equiv 3(\bmod 4) \text { and } k \text { is even; } \\ \frac{3}{2}(k-s)-\frac{s-3}{4} & \text { if } s \equiv 3(\bmod 4) \text { and } k \text { is odd } \\ & \text { or } s \equiv 1(\bmod 4) \text { and } k \text { is even. }\end{cases}
$$

Since $k-s \geq s$, it follows that in all cases $r_{k, s}$ is an integer lying strictly between 0 and $2(k-s)+1$, so $s \bar{\alpha}-\left(\begin{array}{c}s \\ 2\end{array}\right)$ is not an integer. But the integrality of $s \bar{\alpha}$ is necessary for the existence of a tight design since it is the sum of the zeros of $\Psi_{S}(x)$; therefore there are no tight $2 s$-designs with $v=2 k$.

Now, we are able to justify assuming that $\tau \geq 2$ for nonexistence of tight designs.

Proposition 2.2 Let $s \geq 1$. If there exists a nontrivial tight $2 s$-design with $\tau<2$, then there also exists a nontrivial tight $2 s$-design with $\tau \geq 2$.

Proof Suppose $\mathcal{D}$ is a nontrivial tight $2 s-(v, k, \lambda)$ design with $\tau<2$. This means $k \leq$ $v \leq 2 k-1$ because $v \neq 2 k$ by Lemma 2.1 , and so the complementary $2 s-\left(v, v-k, \lambda^{\prime}\right)$ design of $\mathcal{D}$ is a nontrivial tight $2 s$-design with $\tau \geq 2$.

Incidentally, Bannai and Peterson ruled out the case $\tau=2$, observing that it yields symmetric zeros of $\Psi_{s}$ about their mean $\bar{\alpha}$. This is a key observation.

Proposition $2.3([1,9])$ There does not exist any tight $2 s$-design with $v=2 k+1$.

\subsection{Hermite polynomials}

Let $H_{S}(x)$ denote the normalized Hermite polynomial of degree $s$ defined recursively by $H_{0}(x)=1, H_{1}(x)=x$, and for $s \geq 2$,

$$
H_{S}(x)=x H_{s-1}(x)-(s-1) H_{s-2}(x) .
$$

Furthermore, let $\xi_{i}, i=-\left\lfloor\frac{s}{2}\right\rfloor, \ldots,(0), \ldots,\left\lfloor\frac{s}{2}\right\rfloor$, denote the zeros of $H_{s}(x)$ listed in increasing order. It is easily seen that $\xi_{-i}=-\xi_{i}$ for each $i$. See Appendix A for a 
table of $H_{s}(x)$ and their zeros for $1 \leq s \leq 9$. In particular, for the analytical work in Sect. 3, we will make use of the following known estimates.

\section{Proposition 2.4}

(i) If $s$ is odd and $s \geq 5$, then $\xi_{1}^{2}<\sqrt{3}$.

(ii) If $s$ is even and $s \geq 8$, then $\xi_{2}^{2}-\xi_{1}^{2}<\sqrt{3}$.

(iii) If $s=6$, then $1.0<\frac{\xi_{2}^{2}-\xi_{1}^{2}}{3}<1.1,3.5<\frac{\xi_{3}^{2}-\xi_{1}^{2}}{3}<3.6$, and $3.34634<\frac{\xi_{3}^{2}-\xi_{1}^{2}}{\xi_{2}^{2}-\xi_{1}^{2}}$ $<3.34635$.

Proof Items (i) and (ii) are referenced in Bannai's Proposition 13 and proven on page 126 of [12]. Item (iii) can be verified numerically. See Appendix A. (Note that Bannai's Proposition 13 (iii) actually contains an error.)

A useful identity is

$$
H_{s}^{\prime}(x)=s H_{s-1}(x) .
$$

For later reference we define, again as in [1],

$$
\lambda_{i}=\lambda_{i}(\tau)=\left(1-\frac{2}{\tau}\right)^{2}\left(\frac{\xi_{i}^{2}}{6}-\frac{s-1}{6}\right) .
$$

Informally, Proposition 16 in [1] states that as $\beta \rightarrow \infty$, the zeros $x_{i}$ of $\Psi_{s}(x)$ approach $\bar{\alpha}+\beta \xi_{i}+\lambda_{i}$. That is, when suitably normalized, $\Psi_{s}$ behaves like $H_{s}$ for large $\beta$ and fixed $\tau$.

\subsection{The Smith bound}

We now state a useful result for explicitly finding $\beta_{0}$. Sometimes known as the Smith bound, it is a consequence of the Gershgorin circle theorem.

Theorem 2.5 ([11]) Let $P(z)$ be a monic polynomial of degree $n$ and let $\xi_{1}, \ldots, \xi_{n}$ be distinct points approximating the zeros of $P(z)$. Define the circles

$$
\Gamma_{i}=\left\{z:\left|z-\xi_{i}\right| \leq \frac{n\left|P\left(\xi_{i}\right)\right|}{\left|Q^{\prime}\left(\xi_{i}\right)\right|}\right\},
$$

where $Q(z)$ is the monic polynomial of degree $n$ with zeros $\xi_{1}, \ldots, \xi_{n}$. Then the union of the circular regions $\Gamma_{i}$ contains all the zeros of $P(z)$, and any connected component consisting of just $m$ circles $\Gamma_{i}$ contains exactly $m$ zeros of $P(z)$ for each $1 \leq m \leq n$.

Let $s \geq 1$. For each $i \in\left\{-\left\lfloor\frac{s}{2}\right\rfloor, \ldots,(0), \ldots,\left\lfloor\frac{s}{2}\right\rfloor\right\}$, define the monic degree $s$ polynomial (in $z$ )

$$
G_{s}^{(i)}(z)=\frac{s !}{\beta^{s}\left(\begin{array}{c}
v-s \\
s
\end{array}\right)} \Psi_{s}\left(\bar{\alpha}+\beta z+\lambda_{i}\right),
$$


and put $z_{i}=\left(x_{i}-\bar{\alpha}-\lambda_{i}\right) / \beta$, the zero of $G_{s}^{(i)}(z)$ corresponding to $x_{i}$.

We will see from Propositions 2.6 and 2.7 that the $z_{i}$ are well-approximated by the $\xi_{i}$ as $\beta \rightarrow \infty$, independently of $\tau$.

Proposition 2.6 Let $s \geq 1$. Then

$$
\left|z_{i}-\xi_{i}\right| \leq \frac{\left|G_{s}^{(i)}\left(\xi_{i}\right)\right|}{\left|H_{S-1}\left(\xi_{i}\right)\right|}
$$

Proof Simply apply Theorem 2.5 to the polynomial $G_{S}^{(i)}(z)$, letting $Q(z)=H_{S}(z)$, to get

$$
\left|z_{i}-\xi_{i}\right| \leq \frac{s\left|G_{s}^{(i)}\left(\xi_{i}\right)\right|}{\left|H_{S}^{\prime}\left(\xi_{i}\right)\right|}
$$

The result now follows from (2.4).

2.5 Bounding $G_{s}$ in terms of $\beta$

In the next proposition, it is helpful to think of the $G_{S}^{(i)}\left(\xi_{i}\right)$ as functions of $\beta$ and $\tau$.

Proposition 2.7 Let $s \geq 2$. For each $i \in\left\{-\left\lfloor\frac{s}{2}\right\rfloor, \ldots,(0), \ldots,\left\lfloor\frac{s}{2}\right\rfloor\right\}$, there exist constants $B_{i}, C_{i}$ such that whenever $\beta>B_{i}$,

$$
\left|G_{s}^{(i)}\left(\xi_{i}\right)\right|<\frac{C_{i}}{\beta^{2}}
$$

for all $\tau \geq 2$.

The necessary ingredients for this result were proved in [1], although the bound was not directly stated in this form. Therefore, we omit the proof and instead focus on how to (carefully) obtain $B_{i}$ and $C_{i}$ for small $s$ using some basic computer algebra.

Algorithm 2.8 For fixed $s$ and $i$, we may obtain constants $B_{i}$ and $C_{i}$ in Proposition 2.7 by the following procedure.

1. Using (1.1), substitute (2.1), (2.2), (2.3), and (2.5) into (2.6). To defer floatingpoint precision issues, we first replace $\xi_{i}$ in (2.5) by a symbolic parameter $r$.

2. This results in an expression for $G_{s}^{(i)}(r)$ as a rational function of $\beta$, say

$$
G_{s}^{(i)}(r)(\beta, \tau)=\frac{p(r, \beta, \tau)}{q(\beta, \tau)} .
$$

Here, the denominator is

$$
q(\beta, \tau)=\beta^{s}\left(\begin{array}{c}
v-s \\
s
\end{array}\right)=\beta^{s}\left(\begin{array}{c}
\tau^{4}(\tau-1)^{-2} \beta^{2}+\tau+s-1 \\
s
\end{array}\right) .
$$


3. Observe that $q$ is positive for $\beta>0$ and $\tau \geq 2$, and that a lower bound on $q$ is

$$
\tilde{q}(\beta, \tau)=\frac{1}{s !} \beta^{3 s} \tau^{4 s}(\tau-1)^{-2 s} .
$$

This is obtained by replacing each factor in the falling factorial of (2.7) by $\tau^{4}(\tau-$ $1)^{-2} \beta^{2}$.

4. The numerator $p(r, \beta, \tau)$ is, for general $r$, a polynomial of degree $3 s$ in $\beta$. However, for $r=\xi_{i}$, Proposition 2.7 shows the two top coefficients, namely of $\beta^{3 s}$ and $\beta^{3 s-1}$, vanish. Again, to maintain symbolic algebra, we artificially replace these coefficients by zero and call this polynomial $\tilde{p}(r, \beta, \tau)$.

5. We have

$$
\beta^{2} G_{s}^{(i)}(r)(\beta, \tau) \leq \frac{\beta^{2} \tilde{p}(r, \beta, \tau)}{\tilde{q}(\beta, \tau)} .
$$

Note that for $r=\xi_{i}$, the right hand side is a polynomial in $\beta^{-1}$.

6. Consider the coefficient $\kappa_{j}(r, \tau)$ of $\beta^{3 s-j}$ in $\beta^{2} \tilde{p}(r, \beta, \tau)$. With $r=\xi_{i}$, compute (or upper-bound) the maxima

$$
M_{j}=\sup _{\tau \geq 2} \frac{\left|\kappa_{j}\left(\xi_{i}, \tau\right)\right|}{\frac{1}{s !}(\tau-1)^{-2 s} \tau^{4 s}} .
$$

Then, estimating term-by-term,

$$
\left|\beta^{2} G_{s}^{(i)}\left(\xi_{i}\right)(\beta, \tau)\right| \leq M_{0}+M_{1} \beta^{-1}+M_{2} \beta^{-2}+\cdots
$$

for all $\tau \geq 2$.

7. Construct $B_{i}, C_{i}$ so that $\beta>B_{i}$ implies $M_{0}+M_{1} \beta^{-1}+M_{2} \beta^{-2}+\cdots \leq C_{i}$. Note that with sufficiently large $B_{i}$ and a safe choice of $C_{i}$, it suffices to estimate the first few coefficients $M_{j}$.

We should remark that for small $s$, Algorithm 2.8-even the calculation of all $3 s-1$ coefficient maxima $M_{j}$-is essentially instantaneous on today's personal computers. Moreover, deferring the use of floating-point arithmetic to step 5-when $\tau$ is eliminated-makes our subsequent use of floating-point numbers $M_{j}$ quite mild. Indeed, there is virtually no loss in taking $M_{j}$ as (integer) ceilings of the suprema, so that estimating for $C_{i}$ can be performed in $\mathbb{Q}$.

See Appendix B for the results of this calculation for each $5 \leq s \leq 9$ and all relevant indices $i$.

\subsection{Bounding the zeros}

We are now ready for our main result of this section. This is in Bannai's paper [1], but with no attempt to control $\beta$.

Proposition 2.9 Fix a positive integer $s$ and $i \in\left\{-\left\lfloor\frac{s}{2}\right\rfloor, \ldots,(0), \ldots,\left\lfloor\frac{s}{2}\right\rfloor\right\}$. Put $y_{i}=x_{i}-\bar{\alpha}-\beta \xi_{i}$, where recall $x_{i}$ and $\xi_{i}$ are corresponding roots of $\Psi_{s}$ and $H_{s}$, 
respectively. Let $\epsilon>0$ and define

$$
\widehat{\beta}(i, \epsilon)=\max \left\{B_{i}, \frac{C_{i}}{\epsilon D_{i}}\right\},
$$

where $D_{i}=\left|H_{s-1}\left(\xi_{i}\right)\right|$. Then for all $\beta>\widehat{\beta}$ and all $\tau \geq 2$,

$$
\left|y_{i}-\lambda_{i}\right|<\epsilon \text {. }
$$

Proof Observe that $\left|y_{i}-\lambda_{i}\right|=\beta\left|z_{i}-\xi_{i}\right|$, since

$$
x_{i}=\bar{\alpha}+\beta \xi_{i}+y_{i}=\bar{\alpha}+\beta z_{i}+\lambda_{i} .
$$

The estimate now follows easily from Propositions 2.6 and 2.7.

\section{The case $s \geq 5$}

\subsection{Estimates for large $\beta$}

The goal here is to provide formulas for the smallest $\beta_{0}$ possible (see the end of Sect. 2.1) using the $B_{i}$ and $C_{i}$ constructed in Algorithm 2.8. This task is simplified under the conditions that $B_{i}$ is independent of $i$ and $C_{i}=C_{-i}$. There is no loss of generality in assuming this because we can simply take $\bar{B}$ to be the maximum of the $B_{i}$ and $\overline{C_{i}}=\max \left\{C_{i}, C_{-i}\right\}$, and then redefine each $B_{i}=\bar{B}$ and $C_{i}=C_{-i}=\overline{C_{i}}$. In fact, this is not necessary for our explicit constructions because the constants in Appendix B satisfy the above conditions.

Again, for convenience, we denote $\left|H_{s-1}\left(\xi_{i}\right)\right|$ by $D_{i}$ in the following proofs.

Proposition 3.1 Let $s \geq 5$ be odd.

(i) There exists $\beta_{1}$ such that, whenever $\beta>\beta_{1}$,

$$
\left|y_{1}+y_{-1}-2 y_{0}\right|<1 \text {. }
$$

(ii) There exists $\beta_{2}$ such that, whenever $\beta>\beta_{2}$,

$$
\left|y_{i}+y_{-i}-y_{i-1}-y_{-(i-1)}\right|<1+\frac{\xi_{i}^{2}-\xi_{i-1}^{2}}{\xi_{i-1}^{2}-\xi_{i-2}^{2}}\left|y_{i-1}+y_{-(i-1)}-y_{i-2}-y_{-(i-2)}\right|
$$

for $2 \leq i \leq\left\lfloor\frac{s}{2}\right\rfloor$.

(iii) There exists $\beta_{0}(s)$ such that, whenever $\beta>\beta_{0}(s)$ and $y_{i}+y_{-i}-y_{i-1}-y_{-(i-1)}$ is an integer for $1 \leq i \leq\left\lfloor\frac{s}{2}\right\rfloor$, it is necessarily the case that $y_{i}+y_{-i}-y_{i-1}-$ $y_{-(i-1)}=0$ for each $i$. 


\section{Proof}

(i) Observe that since $\tau \geq 2$,

$$
0 \leq 2\left(\lambda_{1}-\lambda_{0}\right)=\left(1-\frac{2}{\tau}\right)^{2} \frac{\xi_{1}^{2}}{3}<\frac{\xi_{1}^{2}}{3} .
$$

Define

$$
\epsilon_{0}=\frac{1}{2}\left(1-\frac{\xi_{1}^{2}}{3}\right)\left(1+\frac{C_{1} D_{0}}{C_{0} D_{1}}\right)^{-1} \text { and } \beta_{1}=\widehat{\beta}\left(0, \epsilon_{0}\right) .
$$

If $\epsilon_{1}=\epsilon_{0} \frac{C_{1} D_{0}}{C_{0} D_{1}}$, then

$$
\widehat{\beta}\left(1, \epsilon_{1}\right)=\beta_{1} \quad \text { and } \quad 2 \epsilon_{0}+2 \epsilon_{1}=1-\frac{\xi_{1}^{2}}{3} .
$$

Hence for $\beta>\beta_{1}$,

$$
\begin{aligned}
\left|y_{1}+y_{-1}-2 y_{0}-2\left(\lambda_{1}-\lambda_{0}\right)\right| & \leq\left|y_{1}-\lambda_{1}\right|+\left|y_{-1}-\lambda_{-1}\right|+2\left|y_{0}-\lambda_{0}\right|, \\
& <2 \epsilon_{0}+2 \epsilon_{1}=1-\frac{\xi_{1}^{2}}{3}
\end{aligned}
$$

By (3.1),

$$
-\left(1-\frac{\xi_{1}^{2}}{3}\right)<y_{1}+y_{-1}-2 y_{0}<1
$$

and the claim follows.

(ii) For $2 \leq i \leq\left\lfloor\frac{s}{2}\right\rfloor$, let

$$
a_{i}=\frac{\xi_{i}^{2}-\xi_{i-1}^{2}}{\xi_{i-1}^{2}-\xi_{i-2}^{2}} \quad \text { and } \quad \epsilon_{i}=\frac{1}{2}\left(1+\left(1+a_{i}\right) \frac{C_{i-1} D_{i}}{C_{i} D_{i-1}}+a_{i} \frac{C_{i-2} D_{i}}{C_{i} D_{i-2}}\right)^{-1}
$$

Note if $2 \leq i \leq\left\lfloor\frac{s}{2}\right\rfloor$, then

$$
\left(\lambda_{i}-\lambda_{i-1}\right)=\left(\lambda_{i-1}-\lambda_{i-2}\right) a_{i} .
$$

Define $\beta_{2}=\max \left\{\widehat{\beta}\left(i, \epsilon_{i}\right): 2 \leq i \leq\left\lfloor\frac{s}{2}\right\rfloor\right\}$. For $\beta>\beta_{2}$ and working as in (i),

$$
\left|y_{i}+y_{-i}-y_{i-1}-y_{-(i-1)}-2\left(\lambda_{i}-\lambda_{i-1}\right)\right|<2 \epsilon_{i}\left(1+\frac{C_{i-1} D_{i}}{C_{i} D_{i-1}}\right) .
$$

Using (3.2) and (3.3) again with $i-1$ replacing $i$,

$$
\begin{aligned}
\mid y_{i}+ & y_{-i}-y_{i-1}-y_{-(i-1)} \mid \\
< & 2 \epsilon_{i}\left(1+\frac{C_{i-1} D_{i}}{C_{i} D_{i-1}}\right)+2 \epsilon_{i} a_{i}\left(\frac{C_{i-1} D_{i}}{C_{i} D_{i-1}}+\frac{C_{i-2} D_{i}}{C_{i} D_{i-2}}\right) \\
& \quad+a_{i}\left|y_{i-1}+y_{-(i-1)}-y_{i-2}-y_{-(i-2)}\right| \\
= & 1+a_{i}\left|y_{i-1}+y_{-(i-1)}-y_{i-2}-y_{-(i-2)}\right|
\end{aligned}
$$


as required.

(iii) Set $\beta_{0}(s)=\max \left\{\beta_{1}, \beta_{2}\right\}$ and assume that $\beta>\beta_{0}(s)$ and $y_{i}+y_{-i}-y_{i-1}-$ $y_{-(i-1)}$ is an integer for $1 \leq i \leq\left\lfloor\frac{s}{2}\right\rfloor$. By (i), $y_{1}+y_{-1}-2 y_{0}=0$ since it is an integer whose absolute value is less than 1 . Assume that $y_{i-1}+y_{-(i-1)}-$ $y_{i-2}-y_{-(i-2)}=0$ for some $2 \leq i \leq\left\lfloor\frac{s}{2}\right\rfloor$. Then (ii) gives the result that $\mid y_{i}+$ $y_{-i}-y_{i-1}-y_{-(i-1)} \mid$ is also less than one and hence equal to 0 since it is an integer, so by induction $y_{i}+y_{-i}-y_{i-1}-y_{-(i-1)}=0$ for $1 \leq i \leq\left\lfloor\frac{s}{2}\right\rfloor$, and so the proof is complete.

Proposition 3.2 Let $s \geq 8$ be even.

(i) There exists $\beta_{1}$ such that, whenever $\beta>\beta_{1}$,

$$
\left|y_{2}+y_{-2}-y_{1}-y_{-1}\right|<1 \text {. }
$$

(ii) There exists $\beta_{2}$ such that, whenever $\beta>\beta_{2}$,

$$
\left|y_{i}+y_{-i}-y_{i-1}-y_{-(i-1)}\right|<1+\frac{\xi_{i}^{2}-\xi_{i-1}^{2}}{\xi_{i-1}^{2}-\xi_{i-2}^{2}}\left|y_{i-1}+y_{-(i-1)}-y_{i-2}-y_{-(i-2)}\right|
$$

for $3 \leq i \leq\left\lfloor\frac{s}{2}\right\rfloor$.

(iii) There exists $\beta_{0}(s)$ such that, whenever $\beta>\beta_{0}(s)$ and $y_{i}+y_{-i}-y_{i-1}-y_{-(i-1)}$ is an integer for $2 \leq i \leq\left\lfloor\frac{s}{2}\right\rfloor$, it is necessarily the case that $y_{i}+y_{-i}-y_{i-1}-$ $y_{-(i-1)}=0$ for each $i$.

\section{Proof}

(i) Since $\tau \geq 2$,

$$
0 \leq 2\left(\lambda_{2}-\lambda_{1}\right)=\left(1-\frac{2}{\tau}\right)^{2} \frac{\xi_{2}^{2}-\xi_{1}^{2}}{3}<\frac{\xi_{2}^{2}-\xi_{1}^{2}}{3} .
$$

Define

$$
\epsilon_{1}=\frac{1}{2}\left(1-\frac{\xi_{2}^{2}-\xi_{1}^{2}}{3}\right)\left(1+\frac{C_{2} D_{1}}{C_{1} D_{2}}\right)^{-1} \quad \text { and } \quad \beta_{1}=\widehat{\beta}\left(1, \epsilon_{1}\right) .
$$

If $\epsilon_{2}=\epsilon_{1} \frac{C_{2} D_{1}}{C_{1} D_{2}}$, then

$$
\widehat{\beta}\left(2, \epsilon_{2}\right)=\beta_{1} \quad \text { and } \quad 2 \epsilon_{1}+2 \epsilon_{2}=1-\frac{\xi_{2}^{2}-\xi_{1}^{2}}{3} .
$$

Hence for $\beta>\beta_{1}$,

$$
\left|y_{2}+y_{-2}-y_{1}-y_{-1}-2\left(\lambda_{2}-\lambda_{1}\right)\right|<2 \epsilon_{1}+2 \epsilon_{2}=1-\frac{\xi_{2}^{2}-\xi_{1}^{2}}{3} .
$$

By (3.4),

$$
-\left(1-\frac{\xi_{2}^{2}-\xi_{1}^{2}}{3}\right)<y_{2}+y_{-2}-y_{1}-y_{-1}<1
$$

and the claim follows. 
(ii) Define $\epsilon_{i}$ and $\beta_{2}$ in as in the proof of Proposition 3.1(ii), but omit $i=2$.

(iii) Imitate the proof of Proposition 3.1(iii).

In the case $s=6, \frac{\xi_{2}^{2}-\xi_{1}^{2}}{3}>1$. Hence it is impossible to choose a $\beta_{1}$ to guarantee that $y_{2}+y_{-2}-y_{1}-y_{-1}=0$ whenever it is an integer and $\beta>\beta_{1}$. However, combining the integrality requirements of $y_{2}+y_{-2}-y_{1}-y_{-1}$ and $y_{3}+y_{-3}-y_{1}-y_{-1}$ allows us to handle this case.

Proposition 3.3 Let $s=6$. There exists $\beta_{0}(6)$ such that, whenever $\beta>\beta_{0}(6)$ and $\left(y_{2}+y_{-2}-y_{1}-y_{-1}\right),\left(y_{3}+y_{-3}-y_{1}-y_{-1}\right)$ are both integers, it is necessarily the case that $y_{2}+y_{-2}-y_{1}-y_{-1}=y_{3}+y_{-3}-y_{1}-y_{-1}=0$.

Proof Observe

$$
0 \leq 2\left(\lambda_{2}-\lambda_{1}\right)<\frac{\xi_{2}^{2}-\xi_{1}^{2}}{3}<1.1 \text { and } 0 \leq 2\left(\lambda_{3}-\lambda_{1}\right)<\frac{\xi_{3}^{2}-\xi_{1}^{2}}{3}<3.6
$$

by Proposition 2.4(iii). Let $a=\frac{\xi_{3}^{2}-\xi_{1}^{2}}{\xi_{2}^{2}-\xi_{1}^{2}}$ and define

$$
\epsilon_{1}=\frac{1}{2}(a-3)\left(1+a+a \frac{C_{2} D_{1}}{C_{1} D_{2}}+\frac{C_{3} D_{1}}{C_{1} D_{3}}\right)^{-1} \quad \text { and } \quad \beta_{0}(6)=\widehat{\beta}\left(1, \epsilon_{1}\right) .
$$

Then, with

$$
\epsilon_{2}=2 \epsilon_{1}\left(1+\frac{C_{2} D_{1}}{C_{1} D_{2}}\right) \text { and } \epsilon_{3}=2 \epsilon_{1}\left(1+\frac{C_{3} D_{1}}{C_{1} D_{3}}\right)
$$

we have

$$
\begin{gathered}
\epsilon_{2} a+\epsilon_{3}=a-3, \\
0<\epsilon_{2}<(a-3) / a \approx 0.10350, \quad \text { and } \\
0<\epsilon_{3}<a-3 \approx 0.34635 .
\end{gathered}
$$

Assume $\beta>\beta_{0}(6)$. Then $\left|y_{2}+y_{-2}-y_{1}-y_{-1}-2\left(\lambda_{2}-\lambda_{1}\right)\right|<\epsilon_{2}$ implies

$$
y_{2}+y_{-2}-y_{1}-y_{-1} \in\{0,1\} \text {. }
$$

Likewise, $\left|y_{3}+y_{-3}-y_{1}-y_{-1}-2\left(\lambda_{3}-\lambda_{1}\right)\right|<\epsilon_{3}$ implies

$$
y_{3}+y_{-3}-y_{1}-y_{-1} \in\{0,1,2,3\} \text {. }
$$

If $y_{2}+y_{-2}-y_{1}-y_{-1}=0$, then $2\left(\lambda_{2}-\lambda_{1}\right)<\epsilon_{2}$ and so $0 \leq 2\left(\lambda_{3}-\lambda_{1}\right)<\epsilon_{2} a$. Hence $y_{3}+y_{-3}-y_{1}-y_{-1}<\epsilon_{2} a+\epsilon_{3}=a-3 \approx 0.34635$, and so $y_{3}+y_{-3}-y_{1}-y_{-1}=0$.

On the other hand, suppose $y_{2}+y_{-2}-y_{1}-y_{-1}=1$. Then $2\left(\lambda_{2}-\lambda_{1}\right)>1-\epsilon_{2}$ and so $2\left(\lambda_{3}-\lambda_{1}\right)>\left(1-\epsilon_{2}\right) a=a-\epsilon_{2} a$. Hence $y_{3}+y_{-3}-y_{1}-y_{-1}>a-\epsilon_{2} a-\epsilon_{3}=3$, a contradiction to (3.5). 
It follows that $y_{2}+y_{-2}-y_{1}-y_{-1}=y_{3}+y_{-3}-y_{1}-y_{-1}=0$.

To summarize, we have the following reworking of Proposition 17 in [1], but with explicit $\beta_{0}$.

Theorem 3.4 For each $s \geq 5$, there are no tight $2 s$-designs with $\beta>\beta_{0}(s)$.

Proof Suppose $x_{-\left\lfloor\frac{s}{2}\right\rfloor}<\cdots<x_{\left\lfloor\frac{s}{2}\right\rfloor}$ are the intersection numbers of a tight $2 s$-design with $\beta>\beta_{0}(s)$. By Proposition 2.2, we may assume $\tau \geq 2$. Then, since $\xi_{-i}=-\xi_{i}$ and $\lambda_{-i}=\lambda_{i}$, we have $x_{i}+x_{-i}-x_{j}-x_{-j}=y_{i}+y_{-i}-y_{j}-y_{-j}$, and this implies that $y_{i}+y_{-i}-y_{j}-y_{-j}$ is an integer for each $i, j \in\left\{(0), 1,2, \ldots,\left\lfloor\frac{s}{2}\right\rfloor\right\}$. By Propositions 3.1(iii), 3.2(iii) and 3.3, these integers must vanish. Specifically,

Case 1: $s$ odd and $\geq 5$ implies $y_{i}+y_{-i}-y_{i-1}-y_{-(i-1)}=0$ for $1 \leq i \leq\left\lfloor\frac{s}{2}\right\rfloor$.

Case 2: $s$ even and $\geq 8$ implies $y_{i}+y_{-i}-y_{i-1}-y_{-(i-1)}=0$ for $2 \leq i \leq\left\lfloor\frac{s}{2}\right\rfloor$.

Case 3: $s=6$ implies $y_{2}+y_{-2}-y_{1}-y_{-1}=y_{3}+y_{-3}-y_{1}-y_{-1}=0$.

In each case, the $x_{i}$ are symmetric about their arithmetic mean $\bar{\alpha}$. By Proposition 2 in [1], this implies $v=2 k+1$. Proposition 2.3 says this is impossible, and the proof is therefore complete.

\subsection{Searching over small $\beta$}

We now turn to small values of $\beta$, for which the problem becomes finite.

Algorithm 3.5 To exclude tight $2 s$-designs with $\beta \leq \beta_{0}$, we may implement the following steps.

1. Compute $\beta_{0}$ from the $B_{i}, C_{i}$ as in the previous section.

2. By Propositions 2.2 and 2.3, we may restrict attention to $\tau>2$. Since $\alpha=$ $\beta^{2} /\left(1-\frac{1}{\tau}\right)^{2}$, it follows that $\alpha<4 \beta_{0}^{2}$. Now, since $\alpha=\left(s \bar{\alpha}+\left(\begin{array}{l}s \\ 2\end{array}\right)\right) / s$ and $s \bar{\alpha}$ is an integer, we have $\alpha \in \frac{1}{s} \mathbb{Z}$. This gives an explicit finite number of admissible $\alpha$, as Bannai observed in [1].

3. Note that, under the assumption of a tight design, the expression

$$
\left(\begin{array}{l}
s \\
2
\end{array}\right) \alpha\left(\alpha+\frac{2 \alpha \tau-\alpha+2}{\alpha \tau^{2}+\tau+1}\right)
$$

is an integer. This is because Proposition 5 in [1] asserts that the coefficient of $x^{s-2}$ in the monic polynomial $s ! \Psi_{s}(x) /\left(\begin{array}{c}v-s \\ s\end{array}\right)$ is

$$
\left(\begin{array}{l}
s \\
2
\end{array}\right) \alpha\left(\alpha+\frac{2 \alpha \tau-\alpha+2}{\alpha \tau^{2}+\tau+1}\right)+\left(\begin{array}{l}
s \\
3
\end{array}\right)\left(3 \alpha+\frac{3 s-1}{4}\right),
$$

and the latter term is always an integer.

4. Fix $\alpha$ as in Step 2. Put $n=k-s=\alpha \tau$ and define

$$
g_{\alpha}(n):=\left(\begin{array}{l}
s \\
2
\end{array}\right) \alpha^{2}\left(1+\frac{2 n-\alpha+2}{n^{2}+n+\alpha}\right)
$$


as in (3.6). As $\tau>2$, we may take $n_{\min }(\alpha)=\max \{s,\lfloor 2 \alpha\rfloor+1\}$ as a lower bound for $n$.

5. Since $g_{\alpha}^{\prime}(n)<0$ for all $n \geq n_{\min }(\alpha)$, it suffices to loop on integers $n$ from $n_{\min }(\alpha)$ until $n_{\max }(\alpha)$, where $g_{\alpha}\left(n_{\max }(\alpha)\right) \leq\left\lfloor\left(\begin{array}{l}s \\ 2\end{array}\right) \alpha^{2}\right\rfloor+1$. Any pairs $(k, v)$ which give integral $g_{\alpha}(n)$ are obtained by $k=n+s$ and $v=\frac{n^{2}+n}{\alpha}+2 s-1$.

6. In principle, at this point the zeros of $\Psi_{s}$ for these pairs $(k, v)$ can be analyzed. However, in practice we found it sufficient in all cases to merely see that $\lambda=$ $\left(\begin{array}{l}v \\ s\end{array}\right)\left(\begin{array}{c}k \\ 2 s\end{array}\right) /\left(\begin{array}{c}v \\ 2 s\end{array}\right)$ was never even an integer.

We wrote a $\mathrm{C}$ program that implements Algorithm 3.5 for a given $s$ and $\beta_{0}$, but with an important optimization. For $n$ near $n_{\max }(\alpha),\left|g_{\alpha}^{\prime}(n)\right|$ is very small so it would be inefficient to loop over $n$ in this region. Therefore, the program loops over integer values of $g_{\alpha}(n)$ from $\left\lceil g_{\alpha}\left(n_{\max }(\alpha)\right)\right\rceil$ and checks the integrality of the corresponding $n$ until the derivative becomes larger than a certain threshold (in absolute value), at which point it begins looping over $n$ from $n_{\min }$ to a much smaller $n_{\max }$. The program is available by contacting the authors.

Our calculations of $\beta_{0}$ in step 1 are displayed in Appendix B. We can report that the method succeeds for $5 \leq s \leq 9$, and probably much higher $s$. We have chosen to avoid continued searches for $s>9$ until new ideas are obtained. In particular, it would be interesting if $s \geq s_{0}$ could be excluded for nontrivial tight $2 s$-designs.

Theorem 3.6 For each $5 \leq s \leq 9$, there are no nontrivial tight $2 s$-designs.

\section{The case $s=4$}

The same analytic approach that is successful for $s \geq 5$ fails when $s=4$. We can only guarantee that

$$
0 \leq 2\left(\lambda_{2}-\lambda_{1}\right)<\frac{\xi_{2}^{2}-\xi_{1}^{2}}{3}=\sqrt{8 / 3} \approx 1.63299
$$

when $\tau \geq 2$, and so there does not exist $\beta_{0}$ such that $\left|y_{2}+y_{-2}-y_{1}-y_{-1}\right|<1$ for all $\beta>\beta_{0}$.

However, it is possible to bound $\left|y_{2}+y_{-2}-y_{1}-y_{-1}\right|$ away from 2 . Let

$$
\epsilon_{1}=\frac{1}{2}(2-\sqrt{8 / 3})\left(1+\frac{C_{2} D_{1}}{C_{1} D_{2}}\right)^{-1} \quad \text { and } \quad \beta_{\star}(4)=\widehat{\beta}\left(1, \epsilon_{1}\right) .
$$

Then the existence of a tight 8-design with $\beta>\beta_{\star}(4)$ and $\tau \geq 2$ implies $y_{2}+y_{-2}-$ $y_{1}-y_{-1}=1$ and $2\left(\lambda_{2}-\lambda_{1}\right) \approx 1$, for which

$$
\tau=\frac{v-7}{k-3} \approx \frac{2}{1-\sqrt[4]{3 / 8}} \approx 9.1971905725
$$

We are able to obtain more precise conditions in the following result. 
Proposition 4.1 If there exists a nontrivial tight 8-design with parameters $v$ and $k$, then $k>25,000$ and $f(k, v)=0$, where $f(k, v)$ is as in Appendix C.

Proof We first used Algorithm 3.5 to find that there are no nontrivial tight 8-designs with $\beta \leq \beta_{\star}(4)$. Thus, any tight 8 -design with $\tau \geq 2$ must have $x_{2}+x_{-2}-x_{1}-x_{-1}=$ $y_{2}+y_{-2}-y_{1}-y_{-1}=1$. Consider the monic and root-centered polynomial

$$
F(x)=24 \Psi_{4}(x+\bar{\alpha}) /\left(\begin{array}{c}
v-4 \\
4
\end{array}\right)=x^{4}+p_{1} x^{3}+p_{2} x^{2}+p_{3} x+p_{4} .
$$

By Eq. (15) in [9], we have

$$
\begin{aligned}
& p_{2}=-\frac{5}{2}-\frac{6(k-3)(k-4)(v-k-3)(v-k-4)}{(v-6)(v-7)^{2}}, \\
& p_{3}=\frac{-4(k-3)(k-4)(v-k-3)(v-k-4)(v-2 k+1)(v-2 k-1)}{(v-5)(v-6)(v-7)^{3}}, \\
& p_{4}=\frac{9}{16}+\frac{3}{2} \cdot \frac{(k-3)(k-4)(v-k-3)(v-k-4) g(k, v)}{(v-4)(v-5)(v-6)(v-7)^{4}},
\end{aligned}
$$

where $g(k, v)$ is as in Appendix C. Assuming $x_{2}+x_{-2}-x_{1}-x_{-1}=1$, the roots of $F(x)$ must be $r_{1}-1 / 4, r_{2}+1 / 4,-r_{1}-1 / 4,-r_{2}+1 / 4$ where $r_{1}-1 / 4=x_{1}-\bar{\alpha}$ and $r_{2}+1 / 4=x_{2}-\bar{\alpha}$ (note that $\left.r_{1}, r_{2} \in \frac{1}{4} \mathbb{Z}\right)$. Expanding,

$$
\begin{aligned}
x^{4} & +p_{2} x^{2}+p_{3} x+p_{4} \\
& =x^{4}+\left(-\frac{1}{8}-r_{1}^{2}-r_{2}^{2}\right) x^{2}+\left(\frac{r_{1}^{2}}{2}-\frac{r_{2}^{2}}{2}\right) x+\left(r_{1}^{2}-\frac{1}{16}\right)\left(r_{2}^{2}-\frac{1}{16}\right),
\end{aligned}
$$

which yields

$$
p_{4}=\left(\frac{p_{2}}{2}+\frac{1}{8}\right)^{2}-p_{3}^{2} .
$$

Substituting (4.1) into (4.2) results in the equation $f(k, v)=0$.

It is possible to apply the Mordell-Weil Theorem to the Diophantine equation $f(k, v)=0$ and obtain a crude finiteness result. We omit the details. In any case, some very recent work of Ziqing Xiang has used careful estimates on the curve $f(k, v)=0$ to completely rule out the existence of nontrivial tight 8-designs.

Acknowledgements The authors would like to thank Jane Wodlinger for helpful discussions. Research of the authors is supported by NSERC. 
Appendix A: Hermite polynomials $H_{s}(x)$ and their zeros, $1 \leq s \leq 9$

\begin{tabular}{ll}
$s$ & $H_{S}(x)$ \\
\hline 1 & $x$ \\
2 & $x^{2}-1$ \\
3 & $x^{3}-3 x$ \\
4 & $x^{4}-6 x^{2}+3$ \\
5 & $x^{5}-10 x^{3}+15 x$ \\
6 & $x^{6}-15 x^{4}+45 x^{2}-15$ \\
7 & $x^{7}-21 x^{5}+105 x^{3}-105 x$ \\
8 & $x^{8}-28 x^{6}+210 x^{4}-420 x^{2}+105$ \\
9 & $x^{9}-36 x^{7}+378 x^{5}-1260 x^{3}+945 x$
\end{tabular}

Note: In the following table, the values of $D_{i}=\left|H_{s-1}\left(\xi_{i}\right)\right|$ are rounded down.

\begin{tabular}{|l|l|l|c|}
\hline$s$ & $i$ & $\xi_{i}$ for $H_{S}(x)$ & $D_{i} \geq$ \\
\hline \multirow{2}{*}{1} & 0 & 0 & 1 \\
\hline 2 & 1 & 1 & 1 \\
\hline \multirow{2}{*}{3} & 0 & 0 & 1 \\
\cline { 2 - 4 } & 1 & $\sqrt{3}$ & 2 \\
\hline \multirow{2}{*}{4} & 1 & $\sqrt{3-\sqrt{6}}=0.7420$ & 1.817 \\
\cline { 2 - 4 } & 2 & $\sqrt{3+\sqrt{6}}=2.3344$ & 5.718 \\
\hline \multirow{4}{*}{5} & 0 & 0 & 3 \\
\cline { 2 - 4 } & 1 & $\sqrt{5-\sqrt{10}}=1.3556$ & 4.649 \\
\cline { 2 - 4 } & 2 & $\sqrt{5+\sqrt{10}}=2.8570$ & 20.64 \\
\hline \multirow{2}{*}{6} & 1 & 0.61670659019 & 6.994 \\
\cline { 2 - 4 } & 2 & 1.88917587775 & 15.02 \\
\cline { 2 - 4 } & 3 & 3.32425743355 & 88.46 \\
\hline
\end{tabular}

\begin{tabular}{|l|l|l|c|}
\hline$s$ & $i$ & $\xi_{i}$ & $D_{i} \geq$ \\
\hline \multirow{7}{*}{7} & 0 & 0 & 15 \\
\cline { 2 - 4 } & 1 & 1.1544 & 20.69 \\
\cline { 2 - 4 } & 2 & 2.3668 & 57.82 \\
\cline { 2 - 4 } & 3 & 3.7504 & 433.1 \\
\hline \multirow{4}{*}{8} & 1 & 0.5391 & 41.09 \\
\cline { 2 - 4 } & 2 & 1.6365 & 73.30 \\
\cline { 2 - 4 } & 3 & 2.8025 & 255.7 \\
\cline { 2 - 4 } & 4 & 4.1445 & 2365 \\
\hline \multirow{3}{*}{9} & 0 & 0 & 105 \\
\cline { 2 - 4 } & 1 & 1.0233 & 135.4 \\
\cline { 2 - 4 } & 2 & 2.0768 & 299.5 \\
\cline { 2 - 4 } & 3 & 3.2054 & 1267 \\
\cline { 2 - 4 } & 4 & 4.5127 & 14159 \\
\hline
\end{tabular}

Appendix B: Constants $B_{i}$ and $C_{i}$ obtained from Algorithm 2.8 and values of $\beta_{0}(s)$

Notes: For convenience, $B_{i}$ was chosen independently of $i$ and $C_{i}$ was taken with $C_{i}=C_{-i}$. 


\begin{tabular}{|c|r|l|r|r|}
\hline$s$ & \multicolumn{1}{|c|}{$B_{i}$} & $C_{i}, i=(0), 1, \ldots,\left\lfloor\frac{s}{2}\right\rfloor$ & $\beta_{0}(s)$ & $\beta_{\star}(s)$ \\
\hline 4 & 10 & 2,14 & & 19.35 \\
5 & 10 & $1,12,88$ & 33.76 & \\
6 & 100 & $11,63,558$ & 156.96 & \\
7 & 10 & $6,93,458,4649$ & 86.55 & \\
8 & 100 & $100,501,2561,30779$ & 106.77 & \\
9 & 100 & $9,773,3186,17732,247789$ & 146.37 & \\
\hline
\end{tabular}

Appendix C: The $f(k, v)$ and $g(k, v)$ used in Proposition 4.1

$$
\begin{aligned}
f(k, v)= & -3408102864+1506333312 k^{2}+974873344 k^{4}-488998144 k^{6} \\
& +62323584 k^{8}-3309568 k^{10}+65536 k^{12}+9310949028 v \\
& -1506333312 k v-4733985888 k^{2} v-1949746688 k^{3} v-1015706784 k^{4} v \\
& +1466994432 k^{5} v+511604992 k^{6} v-249294336 k^{7} v-49810560 k^{8} v \\
& +16547840 k^{9} v+1744896 k^{10} v-393216 k^{11} v-16384 k^{12} v \\
& -11097146016 v^{2}+4733985888 k v^{2}+6922441360 k^{2} v^{2} \\
& +2031413568 k^{3} v^{2}-1428764528 k^{4} v^{2}-1534814976 k^{5} v^{2} \\
& +209662720 k^{6} v^{2}+199242240 k^{7} v^{2}-21567744 k^{8} v^{2}-8724480 k^{9} v^{2} \\
& +786432 k^{10} v^{2}+98304 k^{11} v^{2}+7281931941 v^{3}-5947568016 k v^{3} \\
& -4944873072 k^{2} v^{3}+412538336 k^{3} v^{3}+1856597696 k^{4} v^{3} \\
& +243542016 k^{5} v^{3}-293538048 k^{6} v^{3}-13016064 k^{7} v^{3}+17194752 k^{8} v^{3} \\
& -327680 k^{9} v^{3}-253952 k^{10} v^{3}-2755473732 v^{4}+3929166288 k v^{4} \\
& +1497511456 k^{2} v^{4}-1155170432 k^{3} v^{4}-582955856 k^{4} v^{4} \\
& +183266304 k^{5} v^{4}+58253568 k^{6} v^{4}-16432128 k^{7} v^{4}-1102464 k^{8} v^{4} \\
& +368640 k^{9} v^{4}+544096980 v^{5}-1459281552 k v^{5}+28759472 k^{2} v^{5} \\
& +469164960 k^{3} v^{5}-7038496 k^{4} v^{5}-59703552 k^{5} v^{5}+6536960 k^{6} v^{5} \\
& +2050560 k^{7} v^{5}-328320 k^{8} v^{5}-18769932 v^{6}+293023248 k v^{6} \\
& -127930016 k^{2} v^{6}-58917568 k^{3} v^{6}+27050224 k^{4} v^{6}+1258752 k^{5} v^{6} \\
& +27560816 k^{2} v^{7}-2875616 k^{3} v^{7}-2296192 k^{4} v^{7}+698880 k^{5} v^{7} \\
& -6118466 v^{8}-764688 k v^{8}-1582560 k^{2} v^{8}+772608 k^{3} v^{8}
\end{aligned}
$$




$$
\begin{aligned}
- & 143664 k^{4} v^{8}+10752 k^{5} v^{8}-191952 v^{9}+203472 k v^{9}-52816 k^{2} v^{9} \\
+ & 7520 k^{3} v^{9}-640 k^{4} v^{9}+972 v^{10}-2352 k v^{10}+336 k^{2} v^{10}+45 v^{11} \\
g(k, v)= & 2 k^{4} v-26 k^{4}-4 k^{3} v^{2}+52 k^{3} v+2 k^{2} v^{3}-20 k^{2} v^{2}-120 k^{2} v+258 k^{2} \\
& -6 k v^{3}+120 k v^{2}-258 k v+v^{4}-23 v^{3}+123 v^{2}-433 v+764 .
\end{aligned}
$$

\section{References}

1. Bannai, E.: On tight designs. Q. J. Math. 28, 433-448 (1977)

2. Bremner, A.: A Diophantine equation arising from tight 4-designs. Osaka J. Math. 16, 353-356 (1979)

3. Delsarte, Ph.: An algebraic approach to the association schemes of coding theory. Philips Res. Rep. Suppl. 10 (1973)

4. Enomoto, H., Ito, N., Noda, R.: Tight 4-designs. Osaka J. Math. 16, 39-43 (1979)

5. Ionin, Y.J., Shrikhande, M.S.: $(2 s-1)$-designs with $s$ intersection numbers. Geom. Dedic. 48, 247265 (1993)

6. Ionin, Y.J., Shrikhande, M.S.: Combinatorics of Symmetric Designs. Cambridge University Press, New York (2006)

7. Ito, N.: On tight 4-designs. Osaka J. Math. 12, 493-522 (1975)

8. Lander, E.S.: Symmetric Designs: An Algebraic Approach. London Math. Soc. Lecture Notes, vol. 74. Cambridge University Press, New York (1983)

9. Peterson, C.: On tight 6-designs. Osaka J. Math. 14, 417-435 (1977)

10. Ray-Chaudhuri, D.K., Wilson, R.M.: On $t$-designs. Osaka J. Math. 12, 737-744 (1975)

11. Smith, B.T.: Error bounds for zeros of a polynomial based upon Gerschgorin's theorems. J. ACM 17, 661-674 (1970)

12. Szegö, G.: Orthogonal polynomials. Am. Math. Soc. Colloq. Publ. 23 (1975)

13. van Lint, J.H.: A survey of perfect codes. Rocky Mt. J. Math. 5, 199-224 (1975) 